\title{
Mathematical Explanations and Mathematical Applications
}

\section{Pantsar, Markus}

Springer

2018-12-23

Pantsar , M 2018 , Mathematical Explanations and Mathematical Applications . in B Sriraman (ed.) , Handbook of The Mathematics of The Arts and Sciences . Springer, pp. 1-16. https://doi.org/10.1007/978-3-319-57072-3_30

http://hdl.handle.net/10138/325183

https://doi.org/10.1007/978-3-319-57072-3_30

acceptedVersion

Downloaded from Helda, University of Helsinki institutional repository.

This is an electronic reprint of the original article.

This reprint may differ from the original in pagination and typographic detail.

Please cite the original version. 


\title{
Mathematical explanations and mathematical applications \\ Markus Pantsar, markus.pantsar@gmail.com \\ University of Helsinki
}

\begin{abstract}
One of the key questions in the philosophy of mathematics is the role and status of mathematical applications in the natural sciences. The importance of mathematics for science is indisputable, but philosophers have disagreed on what the relation between mathematical theories and scientific theories are. This chapter presents these topics through a distinction between mathematical applications and mathematical explanations. Particularly important is the question whether mathematical applications are ever indispensable. If so, it has often been argued, such applications should count as proper mathematical explanations.
\end{abstract}

Following Quine, many philosophers have also contended that if there are indispensable mathematical applications in the natural sciences, then the mathematical objects posited in those applications have an independent existence like the scientific objects. Thus the question of mathematical explanations and applications has an important relevance for the ontology of mathematics.

\section{Keywords}

Mathematical applications, mathematical explanations, explanatory power, Quine-Putnam argument, indispensability, mathematical objects, philosophy of mathematics

\section{Introduction}

While the great importance of mathematics for science is generally recognized, there is considerable variation in how that importance is interpreted. It is of course universally accepted 
that there are mathematical applications in science. Already in Antiquity, there was a close connection between mathematics and science. The Pythagoreans held mathematical applications related to many physical phenomena, such as tuning musical instruments, in great importance. For Archimedes, physics and mathematics were closely entwined (Boyer 1989/1991). In modern times, this relationship became even more profound. Since at least Newton's theory of mechanics, mathematical theories have determined to a great degree how theories of physics are formulated. While Newton developed the mathematical theory of calculus concurrently with his theory of mechanics, many of the applications have taken an existing mathematical theory as the framework for a theory of physics. Perhaps the most famous such case was Einstein's application of Riemannian geometry in his general theory of relativity. Wigner (1960) referred to these kinds of "unexpected" applications of mathematical theories when he famously wrote about "the unreasonable effectiveness of mathematics in the natural sciences".

However, to consider the role of mathematics in physics is only the tip of the iceberg. Although the natural sciences are most closely connected to formal mathematical theories, mathematical applications abound also in other disciplines. Due to the development of mathematical theory of probability and statistics, and the central role it plays in any scientific discipline handling empirical data, it is impossible to imagine modern science in a way that does not apply mathematical methods. Since mathematical tools are used in such a universal fashion in scientific explanations, it may perhaps be tempting to jump to the conclusion that also mathematical explanations play an important role in science. Philosophers, however, have stressed the problematic nature of this inference.

The key question in this regard is what the explanatory role of mathematics in the application is. This is often framed as the question whether the application of mathematics has any explanatory power in the scientific explanation, i.e., does it somehow effectively help explain the target phenomenon. It is clear that many mathematical applications do not have proper explanatory power. For example, the explanation that ten protons have ten times the mass of one proton does not explain anything interesting about protons. In a similar vein, explanations based on statistical methods do not appear to have any real explanatory power. For instance, based on a small sample of a population we can apply mathematical tools to find out, say, that a political party has a 24 
percent support (with a margin of error depending on the size of the sample). Clearly the mathematical tools play an important role in making the generalization from the small sample to the whole population. But if we ask what explains the 24 percent support (i.e., why the support is 24 percent) the mathematical theory does not enter the picture. Mathematical tools give us a chance to make generalizations, but the explanatory power is found in the theories and phenomena related to the electorate, not in those mathematical tools. Ultimately, the mathematical tools are in such cases used as a means to save time and effort. In theory, we could survey all members of the population and the statistical tools would not be needed. Thus the mathematical tools could in principle (although not in practice) be removed from the explanation.

As this chapter will show, this idea is central to the question of mathematical explanations in science. If many mathematical applications could be in principle removed from scientific explanations, the natural follow-up question is whether this can be done generally. So the question becomes: are there cases in which mathematical applications are indispensable for scientific explanations? Indispensability can refer to several notions, but here the general idea is that indispensable applications cannot be removed from a scientific explanation without making the explanation unattractive (see Colyvan 1999 for more).

If there are such indispensable applications, it would seem indubitable that mathematical applications have explanatory power in science. In this chapter, the difference between mathematical explanations and mathematical applications is approached based on this distinction between dispensability and indispensability. A dispensable application could (in principle) be removed from the explanation without making the explanation unattractive. An indispensable application, on the other hand, is integral to the explanation and cannot be rmoved. Under this conception, in order for a mathematical application in science to have proper explanatory power, it has to be indispensable. Only in such cases is it possible to talk about mathematical explanations.

It is important to note that indispensability refers here to a strong theoretical criterion. Even if mathematical applications were not indispensable, they can be extremely important or even in scientific practice indispensable. Most philosophers would probably agree that many mathematical applications in science are in fact like that. What philosophers do not agree on is whether this 
practical importance of mathematical applications ever translates into having proper mathematical explanations. This chapter presents several examples of mathematical applications, focusing on the question whether mathematics plays an indispensable explanatory role in them.

The purpose of this chapter is thus not to present a comprehensive account of mathematical applications in science. Instead, the examples are chosen to tease out the difference between mathematical applications and mathematical explanations. It will be seen that this conceptual distinction is not unproblematic. Nevertheless, as becomes clear from the examples below, the distinction can help in getting into terms with the phenomenon of mathematical explanations in science.

\section{Catastrophes and games}

Applied mathematics is a huge field with great importance for many aspects of modern society. Science and industry as we know it would be unimaginable without mathematical applications. As seen in this volume, mathematical applications can be found in science, but also in art, in a wide variety of places - often unexpected ones. The central role of mathematics in the natural sciences is well-documented, but mathematical applications have an equally important place, for example, in modern economics.

While constructing mathematical models is a crucial part of the methodology for many areas of economics, it is important to note that these models are often based on mathematical theories that were not developed for the purposes of economics. One such area is catastrophe theory, which studies the way small changes in the parameters of functions can lead to drastic and sudden changes in the behavior of the functions. Economists got interested in this theory of pure mathematics and saw great potential in applying it to explain economic phenomena. However, these applications turned out to be controversial and as a result catastrophe theory quickly fell out of favor in economics (Rosser Jr 2007).

What happened? In the words of Rosser Jr: 
Sins of intellectual hype and exaggeration were committed as were inappropriate applications of the theory. It is not as widespread in application as its original proponents claimed and is not a general intellectual panacea. (Rosser Jr 2007:3256).

In short, some economists got carried away with the potential of the new application of a mathematical theory. They used mathematically overly simplified and misplaced applications of catastrophe theory, as well as relying too much on qualitative methods (Rosser Jr 2007). Contrast this with another well-known application of a mathematics in economics: game theory. Von Neumann (1928) wrote his famous paper on games to study mathematically the strategies of zerosum games. Morgenstern and von Neumann (1953) took this framework to study economic behavior and it has since developed into a central research paradigm in economics. The mathematical models based on game theory were a novel and highly explanatory development that helped make sense of the behavior of interacting economic agents. Game theory quickly found a wide range of applications and plays a fundamental role in modern economics. (Shubik 1981).

What was the difference? Why is catastrophe theory only slowly regaining some foothold in economics while game theory has been a great success story? Answering such questions conclusively is highly difficult but what should be accounted for is the fact that economists, for a variety of reasons, did not believe that catastrophe theory successfully explained the phenomena it was supposed to explain. But what does it mean to say that a mathematical application explains a scientific phenomenon? Why did one abstract theory of mathematics, game theory, manage to become widely explanatory in economics while another, catastrophe theory, was deemed a failure? Indeed, how can a theory of mathematics explain non-mathematical phenomena in the first place? The problem in answering such questions is that economic phenomena are complex and identifying the reasons for explanatory success of mathematical theories in them can be a daunting prospect. Thus, in order to get to the root of the nature of mathematical explanations, the discussion should first be moved to simpler examples in which the mathematical component of the explanation, as well as the explanatory role it plays, is easier to identify and analyze. This will be done in the examples below.

\section{Curious cicadas and simple strawberries}


Baker (2005) brought an interesting phenomenon from biology (Yoshimura 1997) to the attention of philosophers. There are three species of cicadas that have highly unusual life cycles. Depending on the particular geographical area, the nymphal stage of the cicadas is either 13 or 17 years. It is a biologically interesting question what the advantages of such long nymphal stages are, but for the present context, the key question is why 13 and 17 and not, say, 15 or 16 ? The only feasible explanation that has been proposed so far is based on the mathematical fact that 13 and 17 are prime numbers whereas 15 and 16 are not. Since nymphal periods that are prime numbers have fewer intersections with the periods of other insects with multi-year nymphal stages, the cicadas get an evolutionary advantage. Assuming that there are predators of cicadas with four-year nymphal stages, a 16-year cicada nymphal stage would mean that if the emergence of adult insects coincides at some point, every new generation of cicadas would have to suffer from the presence of those predators. With a 17-year period, however, the next coincidence would be in 68 years. As formulated by Baker, the argument is structured as follows (for the 17-year period):

[1] Having a life-cycle period that minimizes intersection with other (nearby/lower) periods is evolutionarily advantageous. (biological law)

[2] Prime periods minimize intersection (compared to non-prime periods). (number theoretic theorem)

[3] Hence organisms with periodic life cycles are likely to evolve periods that are prime. ('mixed' biological/mathematical law)

[4] Cicadas in ecosystem-type E are limited by biological constraints to periods from 14 to 18 years. (ecological constraint)

[5] Hence cicadas in ecosystem-type E are likely to evolve 17-year periods. (Baker 2009, 614).

The cicadas provide an illuminating case of what kind of explanations in science are considered to be mathematical. Clearly the explanation draws from biological facts (concerning, e.g., nymphal stages), but it is the role of prime numbers that appears to be crucial for the explanation. 
But what kind of an explanation is it? Indeed, while the prime number account of the cicada nymphal stages is clearly an application of mathematics, is it really a mathematical explanation? In the first section of this chapter, the criterion was presented that mathematical explanations can be distinguished from mathematical applications in science due to the indispensable role of mathematics. Do prime numbers play an indispensable role in the cicada case as formulated by Baker? In the next section it will be seen that this is a matter of great debate in the literature. However, on first impression, it is quite understandable to see the cicada nymphal stages as a case of indispensable mathematical application in scientific explanation. In the above formulation, prime numbers seem to have proper explanatory power. If not by referring to the primeness of 13 and 17 , how else can their advantage as nymphal periods over 15 and 16 be explained?

It is important to note that claiming that mathematics plays an indispensable role in the explanation does not amount to claiming that mathematical objects such as prime numbers play a role in the phenomenon. Mathematics can be indispensable for the explanans without mathematical objects being included in the domain of the explanandum. This is an important distinction to make, since the main problem many philosophers have with putatively mathematical explanations - like the cicada prime number nymphal stages - is that they are a bad fit with what explanations in natural sciences are generally thought to be like. Based on such diverse theories of scientific explanation as Salmon (1984) to Woodward (2003), the guiding idea has been that explanations in natural sciences are causal. In modern philosophy of scientific explanations, it is commonplace to accept that scientific facts are explained by finding either their causes (Woodward 2003) or mechanisms (Anderson 2014a, 2014b) responsible for the fact. If we accept this criterion of explanation, the explanatory status of prime numbers in the cicada example becomes highly problematic. After all, prime numbers, like mathematical objects generally, are thought to be abstract and as such (at least according to most philosophers) causally inactive (Rosen 2017).

Clearly this does not bode well for the potential explanatory value of mathematical applications like the one in the cicada case. Insisting on an exclusively causal theory of explanation, combined with the view that abstract objects are causally inactive, would automatically uncover all potential mathematical explanations as merely mathematical applications. In the cicada case, for example, it can be granted that we fruitfully apply arithmetic to a scientific question, but since prime 
numbers cannot play a causal role in the lives of cicadas, this application cannot count as an explanation. A proper explanation is a causal one and involves finding causes or mechanisms responsible for the beginning and the ending of the nymphal stage of the cicada.

Such an explanation could ultimately be preferable for biologists studying cicadas. But even if such a causal explanation were available, would the prime number explanation stop being an explanation? Claiming that the prime number account is not explanatory at all would appear to needlessly limit what can count as an explanation. Perhaps most worryingly, under the causal conception of explanation, all mathematical applications in science would be demoted to the same non-explanatory status. This would be quite problematic, as there seem to be great differences in the status of different mathematical applications. Consider, for example, a situation in which the temperature (rounded to full degrees in centigrade) of each of the ten largest towns in Finland is a prime number. Stating this is clearly applying mathematics, if only on the elementary level of identifying prime numbers. But there is no feasible way that prime numbers could play a role in explaining the temperatures. The primeness of the temperatures is merely a coincidence and, other than noting that there are many primes in common Finnish temperature numbers (e.g., 11, 13, 17, 19), nobody would propose that there is an explanatorily useful pattern of prime numbers involved.

The cicada example, on the other hand, is quite different since the primeness of 13 and 17 plays a key role in the proposed explanation, due to the rare overlapping of the nymphal stages of other insects with multi-year nymphal periods. This way, the prime number periods are not a simple coincidence. Lange (2017) has used this kind of distinction between coincidences and noncoincidences as a criterion of what counts as an explanatory proof in mathematics. If a mathematical theorem is merely a coincidence, its proof has no explanatory value. Only noncoincidental theorems can have explanatory proofs since. A similar distinction could be applicable also to the use of mathematics in science: mathematical explanations in science have to involve non-coincidences. Although they are not causal explanations, if mathematical explanations are not coincidental, they cannot be ruled out as a proper scientific explanation. Lange (2017) calls such non-causal explanations "because without cause" (see also Reutlinger and Saatsi 2018). 
It is not possible to go here into a more detailed discussion on non-causal explanations, but if there are such things, it is clear that mathematical explanations form an important subset of non-causal explanations in science. In many cases, especially in physics, it may indeed be difficult to establish whether an explanation is mathematical or not. One famous example from philosophy is Field's (1980) book Science without Numbers, which aimed to present Newtonian mechanics without using the usual mathematical theory of calculus in it. Whether Field was successful in his project is up to debate, but as Shapiro $(2000,232)$ has commented, many mathematicians feel that Field merely managed to switch from one group of mathematical formalisms to another: while the resulting work may be science without numbers, it is not science without mathematics. Thus the mathematical and physical components are often intertwined in a way that makes it difficult to assess which explanations are characteristically mathematical.

However, there are also cases in which the distinction is easy to make. Lipton (2004) has presented a very simple example of this. Imagine that a bunch of sticks is thrown into the air in a way that makes them turn and twist. Taking a photograph of the sticks as they are falling down, one will notice that many more sticks are in a roughly horizontal position than are in a roughly vertical position. As with the cicadas, a detailed causal explanation is beyond our means. But there is a simple non-causal mathematical explanation: there are many more positions for a stick to be in a horizontal position than there are for it to be in a vertical position (starting from a horizontal position, a stick can be turned around 360 degrees while remaining in horizontal position, while starting the turning from a vertical position it immediately changes into a non-vertical position). Thus a simple mathematical (geometrical) explanation manages to illuminate a physical phenomenon.

Should that case be accepted as a distinctively mathematical explanation? As with the cicadas, there is disagreement. However, it quickly becomes clear that the domain of such explanations is quite extensive. Lange (2013) has illustrated this with a truly simple example. A mother has twenty-three strawberries that she wants to distribute evenly to her three children. Why does she fail? The obvious answer is because twenty-three cannot be divided evenly by three. But is this not already a mathematical explanation? It may not carry the fascination of the cicada example, but the general structure of the explanation is similar. A non-mathematical phenomenon is 
explained by an explanation that is based in an important way on mathematical facts. In both cases, there is a possibility of a causal explanation that does not refer to concepts such as "divisibility". But providing such an explanation, if in practice at all feasible, would not seem to make the mathematical explanation any less powerful as an explanation. Even if there was knowledge of the molecular-level causes or mechanisms that are responsible for the emergence of adult cicadas after the nymphal stages of 13 or 17 years, would the prime number explanation be any less valuable? Similarly, it seems that no amount of physics and chemistry would make the simple arithmetic of the strawberry example less valid as an explanation.

Based on the above examples, it seems clear that there are fruitful explanations of scientific and everyday phenomena that are based directly on mathematical facts. But if we call them mathematical explanations, are we using the term "explanation" in the same sense as with causal explanations? Are there room for both kinds of explanations in science?

\section{What are mathematical explanations like?}

Let us accept for the sake of the argument that there indeed are mathematical explanations in science. The big question is how this could be the case. If mathematical objects are causally inactive and in the natural sciences explanations need to be causal, how can it make sense to have mathematical explanations? In his comprehensive book on the subject, Lange (2017) identifies three types of non-causal scientific explanations: "by constraint", "really statistical" and “dimensional". Of the latter two, "really statistical" explanations form a common family of noncausal explanation (see Lange (2009) for an account of dimensional explanations). For example, let us suppose that a basketball player has a 70 percent success rate in free throws and has completed 19 out of the last 20. From the next 20 free throws, she completes less than 19. Why? There may of course be causal explanations involving the positioning of her arms and other physical factors. But there is also a simple statistical explanation based on regression to the mean. Since completing 19 out of 20 was way above her usual success rate, even without any other information, we should have expected a lower rate with the next 20 attempts. 
While the status of statistical explanations is an interesting question in its own right, neither they nor most dimensional explanations constitute what Lange (2017, 3) calls "distinctively mathematical explanations". Lange contrasts distinctively mathematical explanations with "ordinary explanations employing mathematics", to which statistical explanations belong. Distinctively mathematical explanations are thus not merely explanations that employ mathematics but explanations that show that the explanandum is mathematically necessary. This is what Lange means by explanations "by constraint", which form the most important group of distinctively mathematical explanations. Since explanations by constraint have to be mathematically necessary, the key to them is in the modal information provided by mathematics, which is stronger than the modal information given by the causal mechanisms in the phenomenon:

A distinctively mathematical explanation works by showing the explanandum to be more necessary (given the physical arrangement in question) than ordinary causal laws could render it. (Lange 2013, 485).

It thus becomes clear that the strawberry example is for Lange a distinctively mathematical explanation since it is an explanation by constraint. Mathematics provides the modal information that necessarily 23 strawberries cannot be divided evenly between three children. Whatever the modal information provided by causal explanations of the phenomenon may be, they cannot override this mathematical explanation. Thus mathematical explanations by constraint would retain their role even in cases where there exists a competing explanation that is not distinctively mathematical. Recall, for example, Field's project of constructing science without numbers. For Field (1980, 1989), that is the essential nature of mathematical applications in science: mathematics may be useful in a variety of applications, but ultimately it could be removed from scientific explanations. But explanations by constraint challenge that, as seen from the next example.

There is a classic story in the history of mathematics about Euler and the bridges of Königsberg. In 1736, Königsberg (now Kaliningrad) had seven bridges over the river Pregel. 


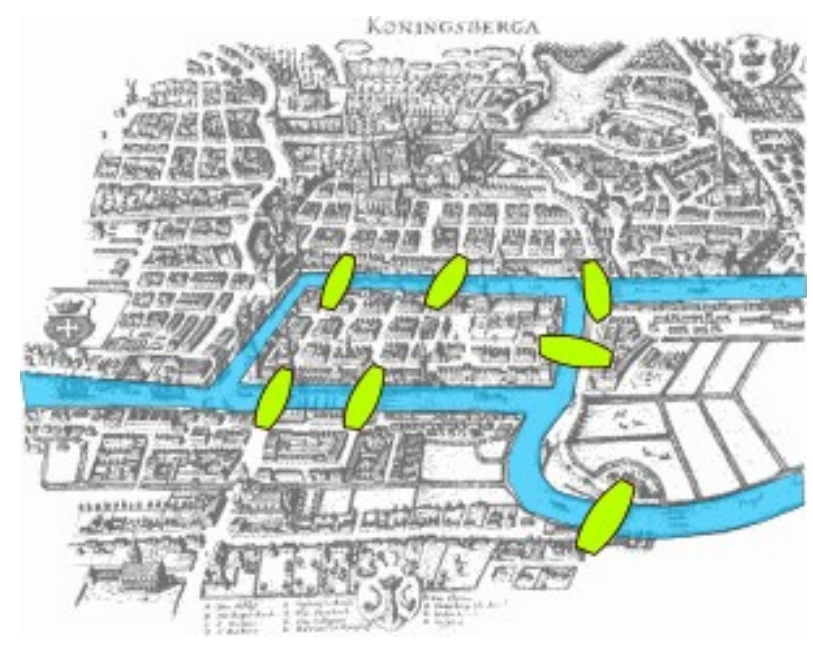

\section{Figure 1: The Bridges of Königsberg (Image: Bogdan Giusca)}

Euler was posed with the following problem: is it possible to walk through the city so that each bridge is crossed exactly once? The solution is famous in the history of mathematics and thought to be the foundation of mathematical graph theory. By distinguishing the relevant factors from the irrelevant, Euler could make the following observations. From any point of a landmass, there needs to be an even number of bridges to another landmass in order for the task to be successful. Since one must both enter and leave the landmass, an uneven number of bridges means that at some point crossing a bridge to the landmass makes it impossible to leave it. The only two exceptions can be the land mass from where the trip begins and the one where it ends. Since in Königsberg there were four landmasses (two islands and the banks of the river), this means that the task is impossible.

In mathematical terms, landmass is simply a node (or vertex) and the bridges are edges. Thus the Königsberg relevant to the problem is merely a graph of four nodes connected by a total of seven edges.

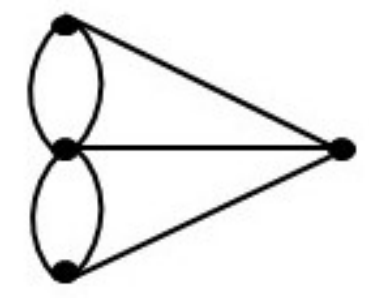

Figure 2: A graph of the Königsberg bridges 
It can now be easily seen that each node has an uneven number (three) of edges. Thus the constraints of the topology of Königsberg explains why the task of crossing each bridge exactly once is impossible. Pincock (2007) has called this kind of explanation abstract, because it appeals primarily to the formal features of a physical system. The physical system of Königsberg in 1736 was not a graph, but the features of it relevant to the bridge problem were entirely formal. Such explanations, Pincock argued against Field, could not be removed from science even if the project of Science without Numbers were successful. The bridges of Königsberg are a clear case of Lange's explanation by constraint. When explaining any actual attempt to cross the bridges, a wide variety of causal factors of different importance would need to be considered. Yet the constraints set by the structure of Königsberg override any such factors, thus making the explanation distinctively mathematical.

In the philosophical literature on the subject, there is much debate on whether cases like the cicada example or the Königsberg bridges should count as mathematical explanations. Criticizing Lange's $(2013,2017)$ account of mathematical explanations, Saatsi $(2011,2016,2018)$ has argued that all the proposed cases of distinctively mathematical explanations can in fact be interpreted as mathematics playing a representational role rather than a proper explanatory role. So in the cicada case, for example, it is due to number theory representing time that we get a seemingly mathematical explanation. Saatsi (2011) calls this kind of relation "knowledge-conferring" and admits that mathematics can justify scientific statements, but not explain them. Under this conception, all putatively mathematical explanations are in fact mathematical applications in the way that Lange calls ordinary scientific explanations employing mathematics, and not proper distinctively mathematical explanations.

\section{Philosophical significance}

The existence and status of mathematical explanations is a hotly debated topic in contemporary philosophy, and the account above has merely managed to scratch the surface. But what exactly is at the stake? Initially, it might seem that the issue is limited to the taxonomy of scientific explanations. But from the taxonomical perspective, the distinctions used above may seem needlessly complicated. How important is it to be able to distinguish between distinctively 
mathematical and ordinary scientific explanations employing mathematics? For taxonomical purposes, a pragmatic approach to such distinctions has considerable appeal. If an explanation in science applies mathematics in a way that is generally seen as important by scientists, it would seem sensible to call it a mathematical explanation, or at least a partly mathematical explanation. Certainly the cicada example, for instance, appears to do just that. In this way, there is a temptation to let scientific practice determine the matter.

Even if such a pragmatic reading of the question of mathematical explanations was not supported, clearly the division of scientific explanations into mathematical and non-mathematical loses relevance if it is considered to be one concerning scientific practice. However, the kind of arguments reviewed above are also relevant for important epistemological and ontological questions. In the philosophical literature, there have been many widely discussed arguments that have depended on the existence and nature of mathematical explanations. Mancosu (2018) identifies two main areas for such arguments. The first is mathematical modelling and idealization in science. The second is the existence of mathematical objects, i.e., the nominalism-platonism debate in the philosophy of mathematics. Due to its central role in understanding the nature of mathematics, this section focuses on the latter.

The indispensability of mathematical explanations has turned out to play a key role in perhaps the most ancient problem in the philosophy of mathematics: do mathematical objects have mindindependent existence. While denials of such existence come in many guises - more or less loosely collected under the name "nominalism" - the defenders of mind-independent mathematical existence are generally referred to as platonists. This may be misleading, as mathematical realism also comes in a variety of different forms, only some of which correspond to Plato's notion of ideal existence. This is not the place to go into that discussion, and in any case, the arguments for realism reviewed in this section do not assume a particular mode of existence for mathematical objects.

The most famous of these arguments comes from Quine $(1951,1966)$ and was developed further by Putnam (1971). As formulated by Putnam: 
[Q]uantification over mathematical entities is indispensable for science, both formal and physical; therefore we should accept such quantification; but this commits us to accepting the existence of the mathematical entities question. [...] Quine [has] stressed both the indispensability of quantification over mathematical entities and the intellectual dishonesty of denying the existence of what one daily presupposes. (Putnam 1971, 347).

Colyvan $(2001,11)$ has formulated the Quine-Putnam indispensability argument as follows:

(1) We ought to have ontological commitment to all and only those entities that are indispensable to our best scientific theories.

(2) Mathematical entities are indispensable to our best scientific theories.

Therefore:

(3) We ought to have ontological commitment to mathematical entities.

As the backdrop for his project of showing that such indispensability of mathematics is in fact not present in science, Field saw the Quine-Putnam argument as the strongest argument for mathematical realism:

The only non-question-begging arguments I have ever heard for the view that mathematics is a body of truths all rest ultimately on the applicability of mathematics to the physical world; so if the applicability to the physical world isn't a good argument either, then there is no reason to regard any part of mathematics is true. (Field 1980, viii)

As noted earlier, Field's project is generally not seen as being successful. However, the indispensability argument has retained an important place in the philosophical literature. But recently the argument has been approached from the explanatory role of mathematics, rather than the quantification over domains of mathematical objects. Indeed, much of the discussion reviewed in the previous sections has been harnessed for an ontological purpose closely resembling that of Quine and Putnam. This was put explicitly by Baker (2009) in his "Enhanced Indispensability Argument" for mathematical realism: 
(1) We ought rationally to believe in the existence of any entity that plays an indispensable explanatory role in our best scientific theories.

(2) Mathematical objects play an indispensable explanatory role in science.

(3) Hence, we ought rationally to believe in the existence of mathematical objects. (Baker 2009, 613).

The subtle, but important enhancement of the Quine-Putnam argument can be seen. It is now also easy to see why the arguments presented in the previous section become relevant for the ontological question concerning mathematical objects, since it is not easy to determine when mathematical applications play a proper explanatory role. In Lange's "distinctively mathematical explanations", the explanatory role of mathematics is putatively indispensable: in the strawberry case, for example, we cannot use the highly informative explanation without employing mathematical concepts of natural numbers and divisibility. Those accepting the Enhanced Indispensability Argument agree that this explanatory role is thus indispensable.

Colyvan (2010) has used the "Kirkwood gaps" as an example of scientific explanation where mathematics carries a significant part of the explanatory power:

The Kirkwood gaps are localized regions in the main asteroid belt between Mars and Jupiter where there are relatively few asteroids. The explanation for the existence and location of these gaps is mathematical and involves the eigenvalues of the local region of the solar system (including Jupiter). The basic idea is that the system has certain resonances and as a consequence some orbits are unstable. Any object initially heading into such an orbit, as a result of regular close encounters with other bodies (most notably Jupiter), will be dragged off to an orbit on either side of its initial orbit. An eigenanalysis delivers a mathematical explanation of both the existence and location of these unstable orbits (Murray and Dermott 2000). It is interesting to note that we can seek out a nonmathematical, causal explanation for why each particular asteroid fails to occupy one of the Kirkwood gaps. Each asteroid, however, will have its own complicated, contingent story about the gravitational forces and collisions that that particular asteroid in question has experienced. Such causal explanations are thus piecemeal and do not tell the whole story. Such explanations do not explain why no asteroid can maintain a stable orbit in the 
Kirkwood gaps. The explanation of this important astronomical fact is provided by the mathematics of eigenvalues (that is, basic functional analysis). We thus have scientific statements involving mathematical entities (the eigenvalues of the system) explaining physical phenomena (the relative absence of asteroids in the Kirkwood gaps). (Colyvan 2010, 302-303).

For Colyvan, the challenge of the nominalist is to deny that in cases like the Kirkwood gaps, mathematical entities play an explanatory role:

If mathematical entities do not play the right sort of role in scientific explanations, then this needs to be spelled out in a way that distinguishes mathematical entities from other entities quantified over in our best scientific theories. (Colyvan 2012, 1043).

This is where Saatsi (2016) sees the major problem with the (Enhanced) indispensability argument. The proponents of the argument have not offered an account of what that "right sort of role" in explanations could be. What he suggests is that the right sort of role for the indispensability argument is not that explanations like the cicada case and the Kirkwood gaps are explanatory; it is whether they are explanatory in an ontologically committing manner (Saatsi 2016). Based on this observation, he proposes a distinction between two types of explanatory role:

Thick explanatory role is played by a fact that bears an ontic relation of explanatory relevance to the explanandum in question.

Thin explanatory role is played by something that allows us to grasp, or (re)present, whatever plays a 'thick' explanatory role. (Saatsi 2016, 1056, italics in the original).

Thus what is required of the mathematical explanation for the Enhanced Indispensability Argument to work is not only that it is indispensable. It needs to be indispensable and play a thick explanatory role. Recall that Saatsi (2011) claimed that the role of prime numbers in the cicada case can be wholly accounted for by the representational role of mathematics, rather than any explanatory role. This distinction can now be seen in terms of thick and thin explanations. Since numbers represent time in the cicada case, the explanatory role is thin. There is no ontic relation involving prime numbers that has explanatory relevance. This way, Saatsi argues that in the kind 
of applications reviewed above the explanatory is always thin and no ontological commitment to mathematical objects is made.

Pincock (2012) has argued in a similar fashion that adding mathematical objects (such as natural numbers) to the explanation adds no explanatory value in examples like those discussed in this chapter. This way, the physical state of affairs which the explanation concerns is seen as orthogonal to whether mathematical objects exist. Regardless of the usefulness of mathematical applications in the natural sciences, the argument goes, the conditions of the physical world neither demand nor preclude the existence of mathematical objects (Yablo 2012).

\section{Conclusion}

In this chapter, the account of mathematical explanations has been studied based on the assumption that distinctively mathematical explanations in science exist if and only if they are indispensable parts of scientific explanation. While this is a widely accepted position among philosophers, it can also be seen as problematic. If the criteria for mathematical explanation are weaker, the matter becomes quite different. Mancosu, for example, has suggested the following definition of mathematical explanations:

mathematical explanation is explanation in natural science carried out by essential appeal to mathematical facts. (Mancosu 2008, 135).

Of course it would first be necessary to determine what "essential appeal" means, but critics like Saatsi would probably claim that Baker's cicada explanation, for instance, is not a mathematical explanation even with this weaker definition. After all, in this case there would be no sharp distinction between the representational and the explanatory role of mathematics, which is the foundation of Saatsi's argument. In the cicada case, number theory can represent time, but Saatsi argues that it plays no explanatory role.

But without a sharp distinction between the representational and the explanatory role of mathematics, things are quite different. Taking our entire scientific-mathematical body of 
knowledge into account, the prime number account of the nymphal stages of the cicadas provides an informative, easily comprehensible explanation that provides immediate justification for accepting the key points in the argument. Given the preference for parsimony in scientific explanations, this is an important aspect of the present topic. Even if there were other methods, they are likely to be much more cumbersome and presumably quite unintuitive for the average scientist. But if indispensability is no longer required, it is natural to include the conglomerate of such factors in the concept of "explanatory power". Following Mancosu's definition, it could then be argued that the explanation with the most explanatory power essentially appeals to mathematical facts, thus making it a mathematical explanation.

This question obviously needs to be studied in more detail, but loosening the criteria from indispensability and the sharp distinction between the representational and the explanatory role of mathematics could help provide us with a concept of explanatory power that is more sensitive to the multitude of factors involved in scientific practice. Such a project would face a challenge in distinguishing between mathematical explanations and mathematical applications that are what Lange calls "really statistical" or which otherwise fail to use mathematics in a proper explanatory role. But if this distinction is feasible in the first place, it should be expected that there are methods available for discerning between the different types of mathematical applications.

The current state of affairs will hardly be the end of the debate on indispensability arguments and the status of mathematical explanations. One could ask, for example, whether Saatsi is presenting an unrealistic criterion above. What could an acceptable ontic relation be for any mathematical fact to play a thick explanatory role? Indeed, it would seem possible to frame all potentially mathematical explanations as explanations through representations. Since the explanandum of natural phenomena do not involve mathematical objects, mathematics is always used to represent something non-mathematical. But this distinction is not unproblematic, since the mathematical theories used in the explanations can play an integral role in characterizing the postulates in the theory, as is the case with calculus and the postulation of continous space-time in Newtonian mechanics. 
Perhaps the future of this debate and the general discussion on mathematical explanations in the natural sciences will move more toward the question what constitutes a mathematical explanation in mathematics, as suggested in Lyon \& Colyvan (2008) (see also Colyvan et al. 2018). If it is not feasible to detach mathematical theories from scientific theories, we should look for theories of explanation that can be used to identify what a mathematical explanation is like without using reference to mathematical objects as a criterion. The nature of mathematical explanations in mathematics is an important topic in the philosophy of mathematics and the question of mathematical explanations in science may need to be studied (at least partly) from that perspective (see Steiner 1978a and 1978b). However, also in that case one must specify what kind of explanatory role is being discussed. Thus studying the question of mathematical explanations independently of the general question of scientific explanations - as philosophers often seem to do - could be misplaced.

\section{Cross references}

N/A

\section{References}

Andersen, H (2014a) A Field Guide to Mechanisms: Part I. Philosophy Compass 9(4):274-283

Andersen, H (2014b). A field guide to mechanisms: Part II. Philosophy Compass 9(4):284-293

Baker, A (2005) Are there Genuine Mathematical Explanations of Physical Phenomena? Mind 114:223-238.

Baker, A (2009) Mathematical Explanation in Science. British Journal for the Philosophy of Science 60:611-33

Boyer, C.B (1989/1991) A History of Mathematics (2nd ed.). Wiley, New York

Colyvan, M (1998) Can the eleatic principle be justified? Canadian Journal of Philosophy 28(3):313-335

Colyvan, M (1999) Confirmation theory and indispensability. Philosophical Studies 96(1):1-19

Colyvan, M (2001) The Indispensability of Mathematics. Oxford University Press, New York

Colyvan, M (2010) There is no easy road to nominalism. Mind 119(474):285-306

Colyvan, M (2012) Road Work Ahead: Heavy Machinery on the Easy Road. Mind 121:1031-46 
Colyvan, M, Cusbert, J, McQueen, K (2018) Two Flavours of Mathematical Explanation. In Reutlinger, A \& Saatsi, J (eds.): Explanation beyond Causation. Oxford University Press, Oxford:231-249

Field, H (1980) Science without Numbers. Princeton University Press, Princeton

Field, H (1989) Realism, Mathematics, and Modality. Blackwell, Oxford

Lange, M (2009) Dimensional explanations. Noûs 43(4):742-775

Lange, M (2013) What Makes a Scientific Explanation Distinctively Mathematical? British Journal for the Philosophy of Science 64:485-511

Lange, M (2017) Because Without Cause: Non-causal Explanations in Science and Mathematics. Oxford University Press, New York

Lipton, P (2004) What Good is an Explanation In: J. Cornwell (ed.), Explanations. Styles of Explanation in Science. Oxford University Press, Oxford:1-21

Lyon, A \& Colyvan, M (2008). The Explanatory Power of Phase Spaces. Philosophia Mathematica $16: 1-17$

Mancosu, P (2008) Mathematical Explanation: Why it Matters. In: Mancosu (ed.): The Philosophy of Mathematical Practice. Oxford University Press, Oxford:134-50

Mancosu, P (2018) Explanation in Mathematics (2018 edition). In: Zalta, E. (ed.): The Stanford Encyclopedia of Philosophy. https://plato.stanford.edu/entries/mathematicsexplanation/\#ExpRolMatSciSomHisRem. Accessed 22 Oct 2018

Morgenstern, O \& Von Neumann, J (1953) Theory of games and economic behavior. Princeton university press, Princeton.

Murray, C.D \& Dermott, S.F (2000) Solar System Dynamics. Cambridge University Press, New York

Pincock, C (2007) A role for mathematics in the physical sciences. Noûs 41(2):253-275

Putnam, H (1971) Philosophy of Logic, Mathematics, Matter and Method. Cambridge University Press, Cambridge (1979):323-357

Pincock, C (2012) Mathematics and Scientific Representation. Oxford University Press, Oxford

Quine, W.V.O (1951) Two Dogmas of Empiricism. In: From a Logical Point of View. Harvard University Press, Cambridge MA 1980:20-46

Quine, W.V.O (1966) The Scope of Language of Science. In: The Ways of Paradox and Other Essays. Random House, New York 1976:215-32 
Reutlinger, A \& Saatsi, J (eds.) (2018) Explanation beyond Causation. Oxford University Press, Oxford

Rosen, G (2017) Abstract objects (2017 edition). In: Zalta, E. (ed.): The Stanford Encyclopedia of Philosophy. https://plato.stanford.edu/entries/abstract-objects. Accessed 22 Oct 2018

Rosser Jr, J. B (2007) The rise and fall of catastrophe theory applications in economics: Was the baby thrown out with the bathwater? Journal of Economic Dynamics and Control 31(10):32553280

Saatsi, J (2011) The Enhanced Indispensability Argument: Representational versus Explanatory Role of Mathematics in Science. British Journal for the Philosophy of Science 62:143-54

Saatsi, J (2016) On the 'indispensable explanatory role of mathematics'. Mind 125(500):1045-70

Saatsi, J (2018) A pluralist account of non-causal explanation in science and mathematics. Metascience 27 (1):3-9

Salmon, W (1984) Scientific Explanation and the Causal Structure of the World. Princeton University Press, Princeton

Shubik, M (1981) Game theory models and methods in political economy. Handbook of Mathematical Economics 1:285-330.

Shapiro, S (2000) Thinking about Mathematics. Oxford University Press, Oxford

Steiner, M (1978a) Mathematical Explanation. Philosophical Studies 34:135-51

Steiner, M (1978b) Mathematics, Explanation, and Scientific Knowledge. Noûs 12:17-28

Wigner, E.P (1960) The unreasonable effectiveness of mathematics in the natural sciences. Communications on Pure and Applied Mathematics 13:291-306

von Neumann, J (1928) Zur Theorie der Gesellschaftsspiele, Math. Ann. 100:295-32

Woodward, J (2003) Making Things Happen: A Theory of Causal Explanation. Oxford University Press, Oxford

Yablo, S (2012) Explanation, extrapolation, and existence. Mind, 121(484):1007-1029

Yoshimura, J (1997) The Evolutionary Origins of Periodical Cicadas during Ice Ages. American Naturalist 49:112-24 